\title{
WIRELESS SYSTEM FOR SLEEPER VIBRATIONS MEASUREMENT
}

This paper describes testing of a wireless technique for measuring the actual sleeper vibrations and determination of their dynamic deflection based on the experimentally acquired acceleration data. Practical measurements of sleeper vibrations and calculation of displacements (deflections) along the track provide insight into the actual condition of layers below sleepers. The main goal was to develop the equipment for structural health monitoring as a basis for the track maintenance management.

Keywords: Railways, track geometry deterioration, vibrations, wireless sensor network.

\section{Introduction}

The track stiffness is a composite value that represents stiffnesses of different materials involved in layers below running rails which support the wheels of vehicles moving along the track. It is defined by combination of stiffnesses of all layers and components of railway superstructure and substructure as follows: rails, resilient pads in the track structure, sleepers, ballast, subballast and subgrade. It represents the proportion between vertical load and elastic rail deflection ( $\mathrm{z}$ ) at a given moment. Track stiffness has long-term influence on track geometry deterioration, deterioration of all components, rail fatigue, noise and vibrations. Low track stiffness causes large rail displacements and large bending moments. As rail displacements increase, growing number of sleepers shares the load, leading to lower forces being exercised on the sleepers. Too low value of track stiffness causes large track settlement, with considerable stress increase in the rails, whereas high global track stiffness leads to small displacement and small bending moments. Thus, fewer sleepers share the load and forces on each sleeper are higher. In addition, dynamic train/track interaction forces are relatively high and can accelerate the process of track deterioration. Therefore, it is important to determine the optimum track stiffness during track design. Apart from determining the optimum global track stiffness, it is necessary to determine optimum stiffness of every single superstructure and substructure element and to adjust them accordingly.

It is important to emphasize that global stiffness of the track changes along a track during its life cycle. Relationship between the load and the deflection is nonlinear due to nonlinearity of stiffness of rail pads and subgrade, as well as the effect of voids beneath sleepers. This means that the track usually gets stiffer with increased loading. Therefore, measurement and analysis of actual values of the vertical track stiffness is of great importance.

The paper presents a wireless system for measurements of actual sleeper vibrations and determination of their dynamic deflection and train speed. This measurement system was developed at the Faculty of Civil Engineering in Belgrade. The goal was to develop the equipment for structural health monitoring as a basis for the track maintenance management.

\section{Characteristics of measuring spot and measuring system}

Measurement was conducted on the railway near thermal power plant "Nikola Tesla" in Obrenovac. This railway serves for coal transport from the surface coalmine "Kolubara" (Fig. 1). Coal transport is delegated to the special railway company "Railway transport TENT". The main purpose of rail transport system is coal transport from surface mine to power plants in Obrenovac and Veliki Crljeni. Railway Obrenovac-Vreoci has $100 \mathrm{~km}$ of track and it transports about 100.000 tonnes of coal per day. It means that this railway handles largest volume of freight in Serbia, and it is one of the busiest railways in Europe. Average traffic on this railway is 60 trains per day (empty and loaded). So far, it has transported more than 800 mil. tonnes of coal (over 25 mil. tonnes per year), and large amounts of other freight, such as the equipment, spare parts, consumable materials, etc. The measurement was conducted during passage of several loaded and empty trains. EDF double hopper wagons (Fig. 1) are used

\footnotetext{
* ${ }^{1}$ Ljiljana M. Brajovic, ${ }^{1}$ Miodrag Malovic, ${ }^{2}$ Zdenka Popovic, ${ }^{2}$ Luka Lazarevic

${ }^{1}$ Department of Mathematics, Physics and Descriptive Geometry, Faculty of Civil Engineering, University of Belgrade, Republic of Serbia

${ }^{2}$ Department of Roads, Airports and Railways, Faculty of Civil Engineering, University of Belgrade, Republic of Serbia

E-mail: ljbrajovic126@gmail.com
} 


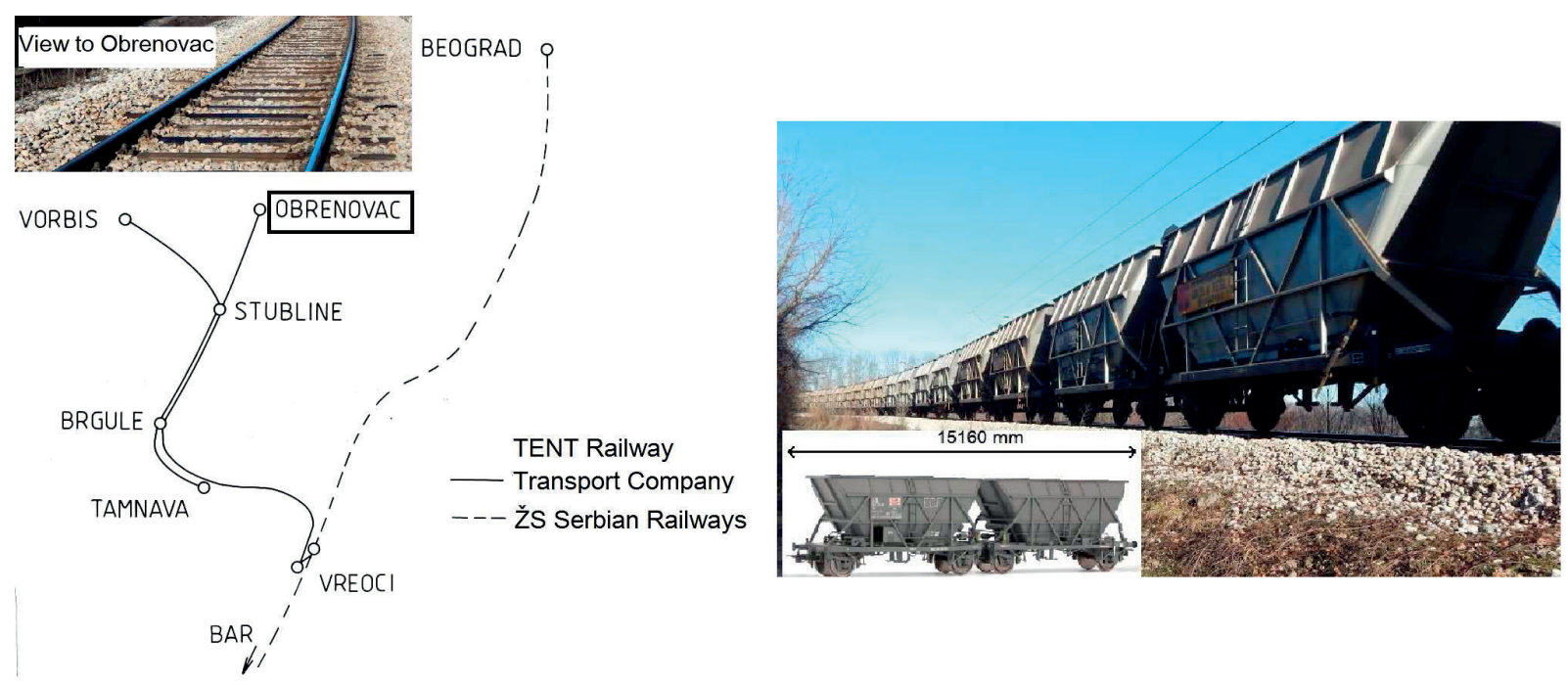

Fig. 1 Rail network Obrenovac-Vreoci and double hopper wagons

for coal transport. Each wagon consists of two hoppers (baskets). Overall wagon length is about $15 \mathrm{~m}$.

Wireless sensor networks are the subject of numerous scientific researches. They are increasingly used in variety of research areas, especially in civil engineering. This paper presents the application of a wireless system for the railway infrastructure measurements. Advantages of wireless sensor networks include cheaper and easier procedures, flexibility and ability to use them in hard to reach places. In addition, there are no expenses for usage of long cables and no practical difficulties that follow their usage. Major advantage of wireless measuring systems is their mobility, and possible relocation of sensor nodes [1].

Need to use wireless technologies is obvious in respect to large areas that railway infrastructure occupies. Condition assessment and monitoring are necessary for all railway infrastructure elements. Large number of infrastructure elements poses a problem that should be investigated, as well as their distribution over a large area [2]. Application of wireless sensor networks for measurements of actual sleeper vibrations and determination of their dynamic deflection is crucial for structural health monitoring. For this purpose, oscillation modes during regular track exploitation, or oscillation modes that are response to artificial excitation are measured and analyzed, or correlation between critical points for given elements is analyzed [3]

A wireless sensor network was developed at the Faculty of Civil Engineering in Belgrade [4] and it consists of wireless sensor nodes and the base station. Sensors are made in surface mount device technology and they use ADuC 845 microcontroller produced by Analog Devices [5]. This processor contains multichannel A/D converter, clock, thermometer, and RS232, I2C and SPI interfaces. It can go into sleep mode with insignificant power consumption. In this state, peripheral components are turned off and only circuits that keep processor in regular working condition are powered. In that case, their power consumption is under $0.4 \mathrm{~mA}$ and the device can work for more than a month with three NiMH batteries, without additional power source (solar cells that are implemented for the purpose of electric recharge - energy harvesting). If solar cells are applied, the device can work almost indefinitely. This depends on the frequency of device wake ups, number of measurements and data transfers performed number of applied solar cells that are connected in parallel in order to create the solar panel, as well as the exposure of the panel to sun rays. Processor speed and wake up period are programmable. As the main sensor, three-axial MEMS accelerometer LIS3LV02DL [6] was used.

There is possibility to use additional analogue sensors with common or separate power supply, and to replace the accelerometer with other digital sensor. The main board can be connected to a display if needed. It is installed into a separate case with the keyboard. The display is irreplaceable in the development phase, and it can serve for device state diagnostics during regular operation. Sensors communicate with the base station (hub) through a radio (RF) modem Decode PRM4 [7]. This modem operates on $863-867 \mathrm{MHz}$ band, which is relatively low frequency that does not support high data flow rate, but it provides wireless communication indoors and in the presence of obstacles, due to better diffraction on larger wavelengths. If longer range is needed, Yagi-Uda antennae may be used, with omnidirectional antenna at the base station. Small stick antennae are used for short ranges. Range of the device with large antennae is up to $1500 \mathrm{~m}$ outdoors. This range can vary a lot depending on whether the device is used in urban environment with high noise level or not. Device block diagram is presented in Fig. 2. As the base station of the system, a PC is used - most commonly a portable laptop. 
A software package that runs under MS Windows operating system was developed to operate the base station. Power reserves of the base station are practically infinite, as well as the memory and processing power. In order to conduct modal analysis of vibrations of civil engineering structures, which is the main purpose of this wireless system, it is necessary to collect all unprocessed signals from multiple wireless devices and to compare them mutually.

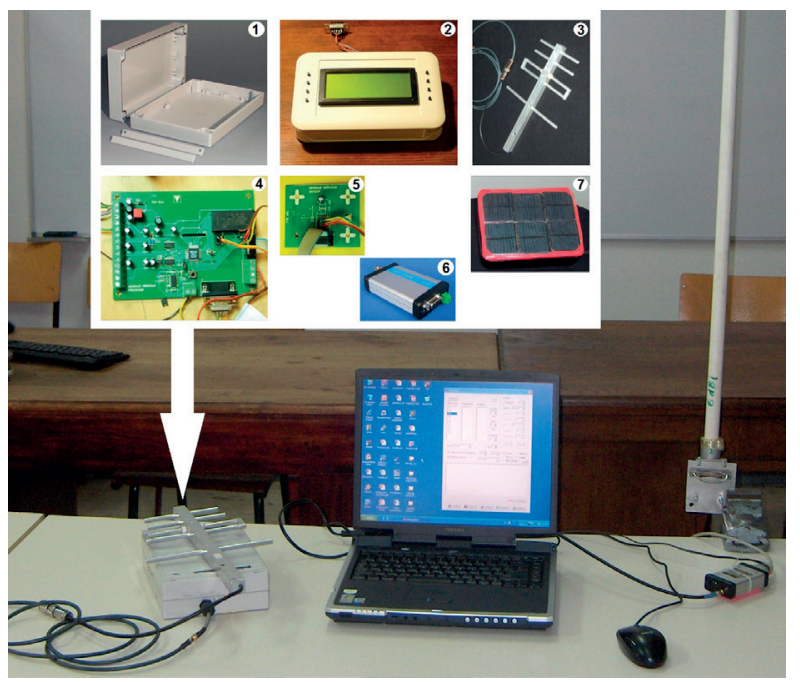

Fig. 2 Sensor node with the hub and device parts: 1) case, 2) display, 3) antenna, 4) main board, 5) accelerometer board,

6) modem and 7) solar cells

The algorithm of system operation implies that sensors are in low power consumption regime most of the time. On the wakeup, sensors spend several seconds checking if there is a signal being broadcasted from the base station. If this signal is detected, sensors remain in stand-by regime for a while expecting a command to begin a measurement, transfer the data, or adjust the parameters. During measurement, 3200 samples of information per axis are collected using variable sampling frequency that ranges from 40 to $2560 \mathrm{~Hz}$. Therefore, time window is between 1.25 and 80 seconds. The data is compressed after the measurement using lossless algorithm of Huffman coding with differential pulse code modulation (DPCM) preprocessor. Since data transfer through a radio connection demands largest power consumption, the goal is to minimize it and to transfer as little bits as possible. The base station conducts data gathering and signal reconstruction. Several methods, including Reference Broadcast Synchronization (RBS), back synchronization [8 and 9], and periodical testing of processors' crystal frequencies and frequency ratios are used to ensure simultaneity of the data collected. Order of magnitude for data synchronization is $0.01 \mathrm{~ms}$ and it exceeds possibilities of mechanical sensors with minimum sampling period of $0.4 \mathrm{~ms}$.
The sampling frequency of $160 \mathrm{~Hz}$ was used and measurement window was $20 \mathrm{~s}$. Three sensors where fixed on railway sleepers on the outer side of the track. Distance between sensors was $3 \mathrm{~m}$. Signal patterns vary from sleeper to sleeper, which depends on the fact whether the passing train is loaded or empty. For loaded trains oscillation amplitudes were smaller, although static deflections were greater. Amplitude of vertical oscillations was about $0.5 \mathrm{~g}$ in case of loaded trains, or about $1 \mathrm{~g}$ in case of empty trains. Exact peak values of acceleration were difficult to measure precisely due to insufficient sampling rate of accelerometers, which was not enough to provide reliable recording of short-term impulses of large forces. Accelerations in other directions were typically lower than vertical acceleration, and they were up to $0.25 \mathrm{~g}$ in case of empty trains. Two diagrams of vertical acceleration as a function of time (for empty trains) are given in Fig. 3 (example 1: regular diagram recorded on a well tamped sleeper; example 2: irregular diagram recorded on a poorly tamped sleeper).

In the first example, a pattern by which wagons are recognizable can be observed. Therefore, it is possible to calculate speed of the train by dividing the wagon length with passage time. Exact wagon length over buffers can vary a bit (in centimeters) due to wagons coupling. Train speed can be estimated with per mille precision. It was assumed that other uncertainties have negligible influence on this calculation. Relative uncertainty of clock frequency of a sensor is $10^{-4}$ in the worst case, and it can go as low as $10^{-6}$ with regular frequency calibrations and application of software correction for temperature changes. Dimension uncertainty due to seasonal variations of temperature and aging is in the order of $10^{-4}$ relative. Uncertainty of digital accelerometer output based on temperature fluctuations does not influence the calculation, because signal amplitude is not related with speed calculation. Loaded train speed of $70.69 \mathrm{~km} / \mathrm{h}$ was determined from the example 1 in Fig. 3.

Even in cases when signal is not visually recognizable, as in the example 2 in Fig. 3, time delay estimation (TDE) technique [10] for determination of train speed can be used. Time domain TDE is performed by observing cross-correlation function of a signal with itself shifted in time. Autocorrelation is a powerful mathematical tool for finding patterns in a signal. It can detect the period of a signal masked by noise or identify fundamental frequency in case it is masked by its harmonics and difficult to detect using other methods such as Fourier transform. In our case, autocorrelation function has its maximum at zero shift, but it should also reach notable value when the signal is shifted by the time needed for a wagon to pass over a sleeper. Figure 4 shows correlation derived using TDE technique for two examples presented in Fig. 3. In the first case, with regular signal, peaks that match the passage of entire EDF wagons are obvious. These peaks appear at distances that are easily observable on the original time domain diagram. Mid-peaks are lower and they have smaller intensity, which perfectly corresponds to the wagon shape - it has four axles, with two mid-axles very close to each other. In 

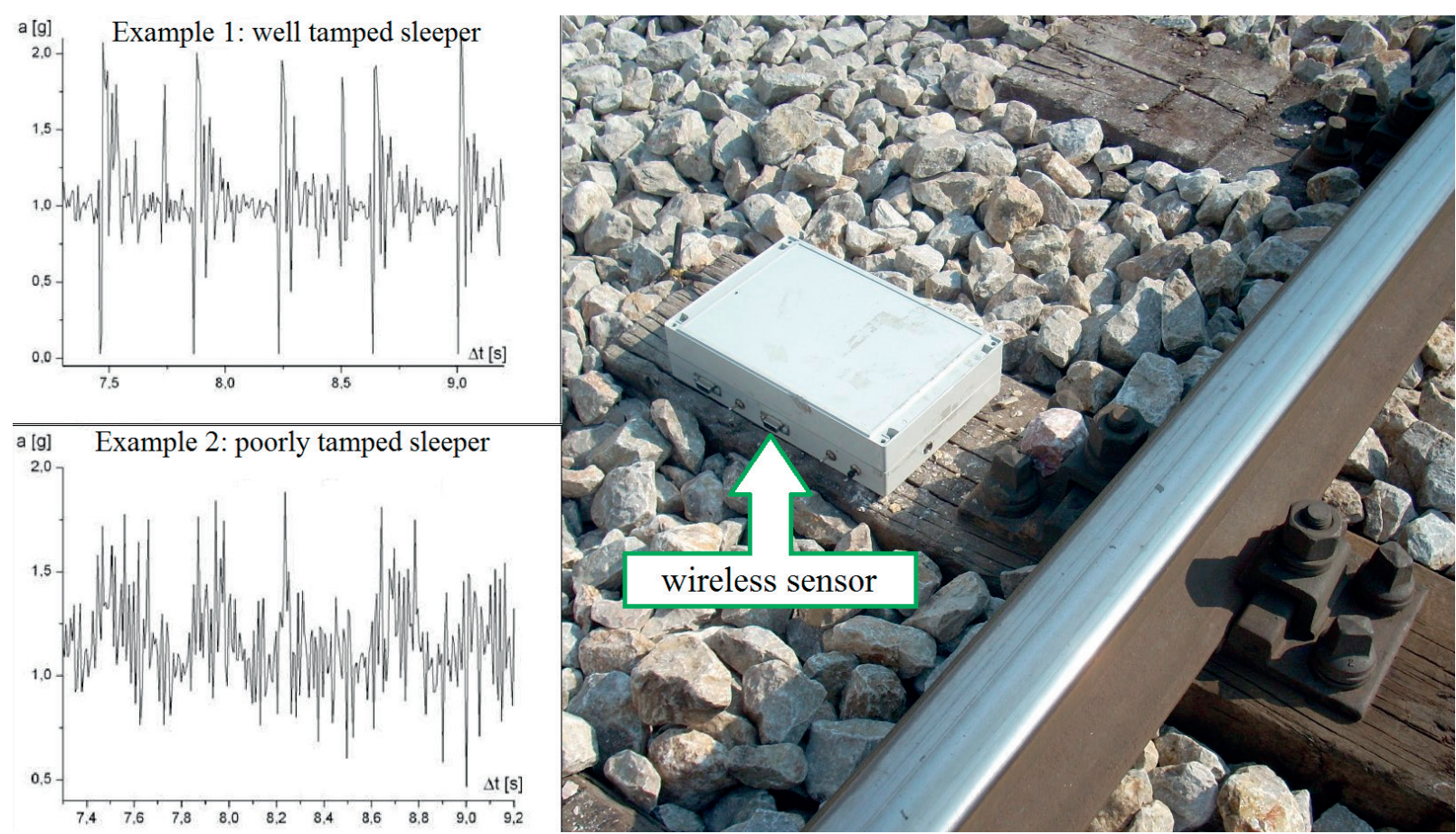

Fig. 3 Vertical acceleration as a function of time on two different sleepers

the second case, with worse quality signal, main peaks and midpeaks cannot be distinguished by the intensity, but the knowledge gained from the previous case enables identification of real main peaks. Final result is similar to the train speed in the first case $-70.97 \mathrm{~km} / \mathrm{h}$. Time delay estimation was performed using 1 ms shift step (and the signal, whose period is $6 \mathrm{~ms}$, was linearly interpolated to fit this). Difference between two calculated speeds corresponds to time offset equal to one third of the sampling period of the accelerometer, or $2 \mathrm{~ms}$.

\section{Potential for dynamic deflection measurement}

Practical measurements of sleeper displacements (deflections) are followed by many difficulties. The main problem is to

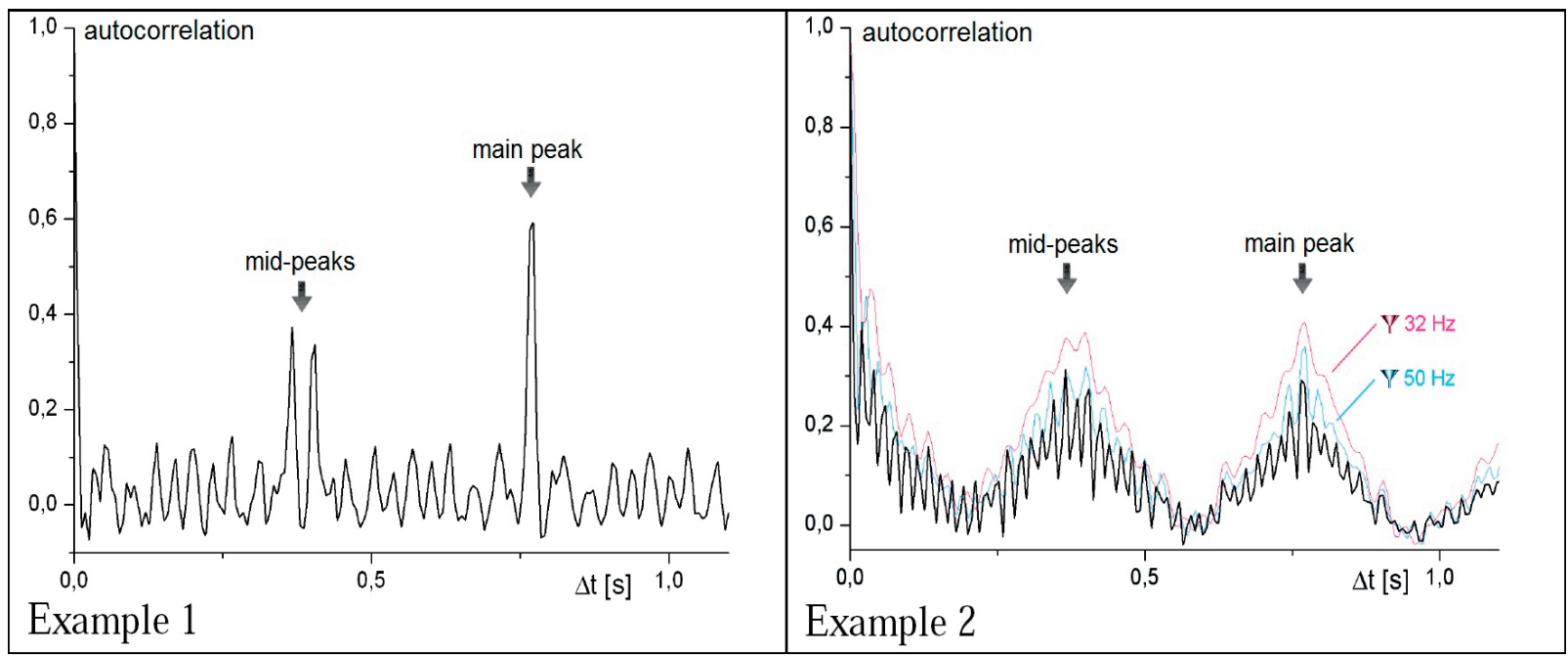

Fig. 4 The result of application of TDE technique on signals from two sleepers 
provide a good reference system because all elements of track superstructure are susceptible to vibrations. Vibrations are also transferred to the surrounding ground where elements of the measuring system have to be placed. Some sensors often used for displacement measurements in other engineering areas are not very efficient in applications on sleepers or other track substructure elements.

If accelerometers are used, there is no need for external reference system because they measure inertial acceleration to which they are subjected. Speed and sleeper position can be determined using single and double integration of an acceleration signal over time, although great difficulties have been found in practice [11]. These difficulties include accumulation of noise and random deviation that rise over time, so software correction methods are necessary. Software corrections were applied over periods needed for sleepers to return into their initial states, which correspond to wagon passages.

The calculation of sleeper displacement by double integration of the signal acquired from described wireless system did not provide completely satisfying results. It turned out that higher sampling frequency is needed in order to record sudden impacts with resolution that is good enough for digital integration.

However, approximate estimation of displacement amplitude of the part of the sleeper where sensor is placed (acceleration and displacement are not uniform along the sleeper length and width) is possible using the existing measurement system. It is not important which points in time we consider as the beginning and end of a period if period value is known. Due to measurement imperfections, which include hysteresis, nonlinearity, noise, and other errors, single and double integration over one period, will not yield exactly zero result, although sleeper speed and displacement should return to their initial values. There are also physical reasons for deviations, such as slower oscillation harmonics of the train and the track and differences between wagons. The correction is performed by shifting direct component of the signal (its mid-level) by unique value for each wagon passage period, to ensure that the integration results in nil vertical displacement.

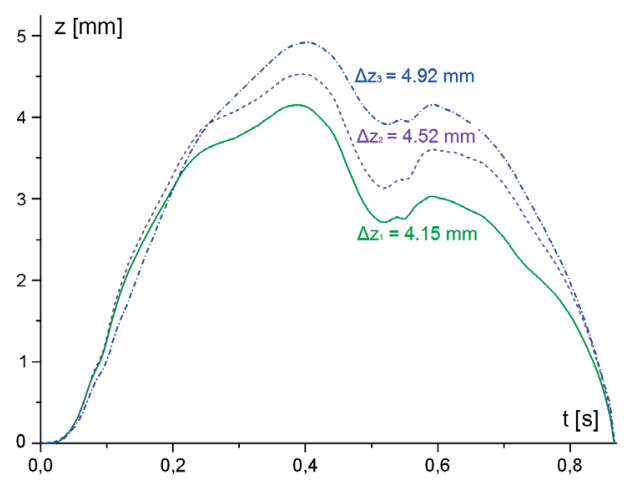

Fig. 5 Examples of calculation of vertical displacement (dynamic deflection) using double integration for the case of regular signal
Figure 5 shows examples of calculation of dynamic vertical displacement (deflection) $z$ of a sleeper using the method of double integration, with correction, for three different periods (wagons). In this case, the train speed was estimated to 62.52 $\mathrm{km} / \mathrm{h}$. Values on diagram axes are relative - reference $z$ value was not determined, and time was counted in respect to the beginning of an arbitrary period. Although differences are significant, the pattern is obvious, and deviation of displacement amplitude in the case of loaded train is in the order of $10 \%$. It can be concluded that displacement amplitude is about $2 \mathrm{~mm}$.

Largest deviations occur for passages of empty trains over sleepers with poor quality (example 2 in Fig. 3). A pattern is not recognizable here and amplitudes vary up to $1: 2.5$. The displacement amplitude is maximum in this case and its value is about $8 \mathrm{~mm}$.

\section{Conclusion}

A wireless system for vibrations measurement, developed at the Faculty of Civil Engineering in Belgrade, was tested on the railway next to the thermal power plant of Obrenovac. It measured vibrations of track sleepers during passage of empty and loaded trains that transport coal. Train speed was determined with good reliability and dynamic deflection of sleepers was approximately estimated based on the experimentally acquired acceleration data. Autocorrelation of acceleration function can serve as a good criterion to estimate quality of sleeper support. Practical measurements of sleeper vibrations and calculation of displacements (deflections) along the track provide insight into the actual condition of layers below sleepers: ballast (including effect of voids beneath sleepers), sub-ballast and subgrade soil. The effective superstructure maintenance management must include analysis of the substructure [12 and 13]. Data that indicates the state of all layers below the sleepers is obtained by measuring the vibrations of track sleepers during the passage of trains. This method of dynamic deflection measurements of sleepers can be a basis for structural health monitoring and the track maintenance management on railway networks with limited length (such as the railway networks of the TENT Transport Company, Fig. 1).

\section{Acknowledgement}

This work was supported by the Ministry of Science and Technological Development of the Republic of Serbia through the research project No. 36012: "Research of technical-technological, staff and organizational capacity of Serbian Railways, from the viewpoint of current and future European Union requirements". 


\section{References}

[1] PUCCINELLI, D., HAENGGI, M.: Wireless Sensor Networks: Applications and Challenges of Ubiquitous Sensing, Circuits and Systems Magazine, IEEE, 5(3), 2005, 19-31.

[2] KAEWUNRUEN, S., M. REMENNIKOV, A.: Trends in Vibration-based Structural Health Monitoring of Railway Sleepers, in R. C. Sapri (ed), Mechanical Vibration: Measurement, Effect and Control, 2009, 3-4.

[3] LYNCH, J. P., LOH, K. J.: A summary Review of Wireless Sensors and Sensor Networks for Structural Health Monitoring, Shock and Vibration Digest, 38(2), 2006, 91-130.

[4] MALOVIC, M., BRAJOVIC, LJ., MISKOVIC, Z., TODOROVIC, G.: Vibration Measurement using Wireless Sensor Network, Tehnika, 66(6), 2012, 883-889.

[5] Analog Devices, ADuc845 microconverter datasheet, from http://www.analog.com/static/imported-files/data_sheets/ ADUC845_847_848.pdf.

[6] ST Microelectronics, LIS3LV02DL accelerometer datasheet, from http://www.st.com/st-web-i/static/active/en/resource/technical/ document/datasheet/ CD00091417.pdf.

[7] Decode, PRM-4 radio modem datasheet, from http://www.decode.rs/documentation/ PRM_4-datasheet.pdf

[8] RHEE, I. K., LEE, J., KIM, J., SERPEDIN, E., WU, Y. C.: Clock Synchronization in Wireless Sensor Networks: An Overview, Sensors, 9(1), 2009, 56-85.

[9] GAUTAM, G. C., SHARMA, T. P.: A Comparative Study of Time Synchronization Protocols in Wireless Sensor Networks, Intern. J. of Applied Engineering Research, 1(4), 2011, 691-705.

[10] CARTER, G. C.: Coherence and Time Delay Estimation: An Applied Tutorial for Research, Development, Test, and Evaluation Engineers. IEEE Press, 1993.

[11] COX, S. J.: Deflection of Sleeper in Ballast, Vehicle System Dynamics Supplement, vol. 24, 1995, 146-153.

[12] PUZAVAC, L., POPOVIC, Z., LAZAREVIC, L.: Influence of Track Stiffness on Track Behaviour under Vertical Load, Promet Traffic \& Transportation, vol. 24, No. 4, 2012, 387-394.

[13] IZVOLT, L., KARDOS, J., LELAK, J., MECAR, M.: Diagnostics of the Track Substructure Model and its Practical use, Communications - Scientific Letters of the University of Zilina, vol. 4, 2005, 31-36. 